\title{
Masculinidad y hombre maltratador ¿pueden las creencias de hombres y mujeres propiciar violencia de género?
}

\author{
Masculinity and abusive man. Can men and women \\ beliefs encourage gender violence?
}

\author{
Efrain Duarte ${ }^{l}$ \\ Jorge Francisco Gómez \\ Carlos David Carrillo ${ }^{3}$
}

Resumen

Esta investigación tuvo como propósito explorar la relación entre las creencias sobre masculinidad y hombre maltratador, incorporando la perspectiva de género como una herramienta para el análisis de dichas creencias, y tomando en cuenta la opinión de mujeres y de hombres con respecto a ellas. El estudio se llevó a cabo en el Estado de Yucatán, México. Participaron 117 mujeres (58.5\%) y 83 hombres (41.5\%). De ellos, 173 (86.5\%) tenían residencia en la zona urbana y 27 (13.5\%) residían en la zona rural. La recolección de datos se realizó a través de dos escalas: la Escala de Creencias acerca de la Masculinidad (ECAM), (Gómez \& Carrillo, 2007) y la Escala de Creencias acerca del Hombre Maltratador (ECHOM), (Gómez, Carrillo \& Revilla, 2008). Todo esto, dentro del contexto de un estudio cuasiexperimental, ex postfacto, transversal, de campo, de dos muestras independientes. Los resultados muestran que existen diferencias en las creencias acerca de la masculinidad y el hombre maltratador, entre las mujeres y los hombres participantes; que las creencias sobre estos dos constructos se relacionan significativamente, encontrando - en las mujeres- mayor relación con el uso de la violencia como consecuencia del discurso y normativa social que se "impone" a los hombres; y, por

\footnotetext{
Efraín Duarte, Lic. en Psicología, Mtro. en Educación Superior, dbricen@uady.mx Jorge Francisco Gómez, Lic. en Psicología, draxonos@hotmail.com

Carlos David Carrillo, Lic. en Psicología, Mtro. en Psicología, cartruji@uady.mx, Universidad Autónoma de Yucatán, Mérida, México.
} 
otro lado, que tanto mujeres como hombres consideran que la violencia es normal que la ejerzan los hombres, aunque ambos la rechazan. Por último, es importante señalar que el constructo de masculinidad está basado en la concepción hegemónica del término, por lo tanto, sería interesante explorar los modelos alternativos de la masculinidad.

Palabras clave: Masculinidad, hombre maltratador, violencia de género, creencias.

\begin{abstract}
This research was aimed to explore the relationship between beliefs about masculinity and abusive man, incorporating a gender perspective as a tool for the analysis of such beliefs and taking into account the views of women and men with regard to those beliefs. It was conducted in the state of Yucatan, Mexico. With a sample of $117(58.5 \%)$ women and 83 (41.5\%) men, 173 (86.5\%) living in urban areas and $27(13.5 \%)$ in rural areas. Data collection was taken through two scales: the Scale of Beliefs about Masculinity (ECAM), (Gomez \& Carrillo, 2007) and the Scale of Beliefs about Abusive Men (ECHOM), (Gomez, Carrillo \& Revilla, 2008). The research is a quasiexperimental, ex post facto, two independent groups, and field study. The outcomes show that there are differences in beliefs about masculinity and about the abusive man among women and men participants, that beliefs about these two constructs are significantly related, finding - in women - the closest relationship to the use of violence as a result of speech and social norms that "imposes" the men and, by other hand, both women and men consider that violence is a normal men's behavior, but both of them reject it. At last, is important to remark that the construct of masculinity is based on the hegemonic conception of the term, therefore, it would be interesting to explore alternative models of masculinity.
\end{abstract}

Key words: Masculinity, abusive man, gender violence, beliefs. 
Definición del problema y perspectiva teórica

Si se analizara y reflexionara acerca del origen de la violencia de género, esta reflexión se tendría que remontar hasta el origen de la civilización tal cual hoy se conoce. La búsqueda llevaría a explorar los procesos de socialización y construcción de identidades en los hombres y mujeres, y la forma en la que estos procesos se fueron introyectando en las diversas culturas.

Durante mucho tiempo la violencia estuvo explicada como un componente inherente a la naturaleza humana e identificada con el varón. Sin embargo, con el paso del tiempo, y gracias a los estudios realizados por el movimiento feminista, se llegó a la conclusión de que la violencia no es innata, sino aprendida, y que se aprende entre otras cosas, a través de un complejo sistema llamado género.

En las tres últimas décadas, en varios países del mundo, se ha impulsado el conocimiento de las diferencias entre hombres y mujeres en áreas como la educación, el empleo, la participación ciudadana, la psicología y la salud, con el propósito de visibilizar y erradicar respectivamente las desigualdades. A lo largo de la historia se puede hallar que esta brecha se fue haciendo cada vez más grande, hasta tener como consecuencia la sobrevaloración del quehacer del hombre y la subvalorización del quehacer de la mujer, donde su espacio se convirtió en privado, silencioso, escondido y secreto. En tanto, el del varón se convirtió en un espacio público, del pensamiento, del poder y más tarde el del ciudadano (Lamas, 1997).

Por extraño que pueda parecer, el espacio de la mujer, el hogar (lugar asociado originalmente con el cariño, la compañía mutua y de satisfacción de necesidades básicas para el ser humano), puede, lejos de ser un lugar de protección y seguridad, convertirse paradójicamente en un sitio de riesgo para el surgimiento de conductas violentas, y apoyados en el mito de que "la ropa sucia se lava en casa", estas conductas quedan en secreto y al mismo tiempo impunes (Corral, 2000).

En la actualidad, para entender las consecuencias que la brecha de género ha traído para hombres y mujeres, resulta importante conocer cómo aprenden los hombres a ser tales, y cómo aprenden las mujeres a 
conceptualizar a los hombres. Este proceso de aprendizaje está implicado en el constructo de masculinidad. De igual manera, resulta importante desentrañar la forma en que se realiza esta pedagogía de género, es decir, cómo se va incorporando una estructura relacional entre los propios hombres y entre las mujeres con respecto a los hombres.

Por otro lado, un aspecto que es importante profundizar con respecto a la masculinidad es la relación que el poder y la violencia va teniendo en la construcción de la identidad de los hombres, y cómo las mujeres conceptualizan hasta cierto punto que algo esencial en el ser hombre o masculino es el hecho de ser violento o abusar del poder. Este proceso de asociación no es casual, ya que hay que recordar que las construcciones de género, al ser construcciones sociales, son compartidas por hombres y mujeres. Por lo tanto, resulta de vital importancia conocer, explorar y analizar la construcción de la masculinidad en las mujeres y los hombres. Estos ejercicios redundarán en una mejor comprensión de la realidad actual y también en la generación de alternativas que promuevan vidas saludables en todos.

Hoy por hoy, la prevención de la violencia familiar es uno de los ejes rectores de políticas públicas en sectores como la educación, la economía, los procesos judiciales y la salud. Sin embargo, estas políticas son consideradas por quienes las ejecutan de una manera vertical, esto es, como un programa o una política más, cuando su consideración debería ser de manera transversal, donde la prevención de la violencia y la perspectiva de género se incluyan en todo el sistema y el orden de gobierno.

Además de políticas públicas, las vías de actuación deben estar presentes en diversos escenarios; a nivel escolar, a través de la educación con equidad y no sexista; a nivel relacional, con el rechazo de la violencia como forma de solución de problemas; a nivel de la atención y prevención, a través de proveer apoyo a las necesidades básicas de quienes viven en situación de violencia; a nivel profesional, con la preparación de profesionales; y a nivel judicial, con acciones más rápidas, efectivas e integrales, que permitan y coadyuven a la erradicación de la violencia (Contreras, 2005).

En este sentido, para poder entender de una manera integral la dinámica de la violencia de género, es necesario, sin que esto signifique re- 
dundar, analizar la construcción genérica. Esta construcción establece cierta normatividad en la conducta de las mujeres y los varones. La normatividad se encuentra enraizada en los discursos sociales, en las prácticas y en las instituciones que se encargan de socializar y de construir a hombres y a mujeres.

Si bien la investigación en cuanto al tema de género ha transitado a lo largo del tiempo por una serie de cambios y de estructuraciones, hoy se entiende que hablar de género no es hablar única y exclusivamente de mujeres. Uno de los grandes aportes de los estudios de género radica en el espacio otorgado a la reflexión, construcción, deconstrucción y reconstrucción de lo que significa ser hombre y ser mujer en la sociedad. En la actualidad, hablar de género es hablar de mujeres y de hombres.

Desde esta perspectiva, resulta de vital importancia explorar el concepto y las creencias acerca de la Masculinidad y del Hombre Maltratador, que poseen tanto hombres como mujeres. Además, es importante utilizar la perspectiva de género como una herramienta que permita analizar la forma en la que estas construcciones impactan en las dinámicas relacionales entre las mujeres y los hombres.

En este sentido, la investigación alrededor de esta temática resulta fundamental, considerando que el conocimiento científico con perspectiva de género es una herramienta útil en los esfuerzos que se llevan a cabo para prevenir y erradicar la violencia doméstica en contra de las mujeres, así como el medio más efectivo para llegar a una comprensión integral del problema en México (Contreras, 2005).

Algunos esfuerzos ya realizados han logrado generar información sobre el tema como: la identificación de espacios donde se perpetra la violencia; que ésta se ejerce por individuos conocidos hacia quienes se siente o se tiene una vinculación afectiva; y que, en la mayoría de los casos, el agresor es un varón (padre, esposo, hermano, pareja, colega), (Primer Informe Mundial sobre Violencia y Salud, 2003).

En México, como en otras naciones del mundo, miembros de la Organización Mundial de la Salud (OMS), se llevaron a cabo acciones que permitieron obtener información acerca de la situación de la violencia hacia las mujeres en el país. En el año 2003 se aplicaron dos instrumentos: la Encuesta Nacional de Violencia Contra las Mujeres (ENVIM) y la En- 
cuesta de la Dinámica de las Relaciones en los Hogares (ENDIREH), cuya última actualización se realizó en 2006.

En Yucatán, según la ENVIM (2003), 26.0\% de las mujeres había vivido violencia con su pareja actual, el 34.8\% reconoció haber sufrido violencia de pareja alguna vez en su vida, el 13.2\% había sufrido violencia durante el embarazo, 15.7\% había sufrido violencia sexual y el $59.7 \%$ reconocía haber sufrido violencia alguna vez en su vida. Algunas de estas cifras se encuentran arriba de las medias nacionales y las que no, se encuentran en una ligera cercanía, lo cual puede dar una idea de la gravedad de la situación en dicho estado. En este mismo sentido, según la ENDIREH (2003), el 34\% de las mujeres había sufrido algún tipo de violencia. Los tipos de violencia que explora la encuesta son: la sexual, la física, la económica y la emocional o psicológica. El rango de tiempo de la medición se realiza alrededor de los últimos 12 meses anteriores a la administración de la encuesta.

Otro aspecto importante a considerar dentro de este contexto es el hecho de que, hasta ahora, la mayoría de las investigaciones han tomado únicamente a las mujeres como población objeto de estudio, siendo que son precisamente los hombres quienes, en la mayoría de los casos, ejercen la violencia. El hecho de no incorporar el punto de vista masculino da como resultado una mirada parcial y una perspectiva incompleta respecto al problema. La complejidad de la violencia entre parejas demanda nuevas aportaciones para el avance de su entendimiento. La presente investigación tiene como uno de sus principales aportes el incorporar la visión de los hombres sobre su propio ejercicio de la violencia, con lo cual pretende ser un eslabón más en el esfuerzo por combatir la problemática.

Así, para poder realizar este trabajo fue necesario contar con dos escalas que midieran las creencias acerca de la masculinidad y del hombre maltratador, considerando que las creencias de las personas acerca de lo que significa ser hombre y ser hombre maltratador pueden convertirse en un factor de riesgo. Identificar el ser hombre con el hecho de ser maltratador, puede llevar a dos fenómenos: primero, que las mujeres y los hombres no identifiquen la violencia que viven o que ejercen, o que la minimicen o justifiquen; y segundo, que los hombres y las mujeres consideren como parte 
de la identidad masculina el uso de la violencia y por lo tanto sea visto como algo "normal" y "natural" de ser masculino o de ser hombre, lo cual normaliza y naturaliza su uso y abuso dentro de la relaciones entre individuos.

Al mismo tiempo, que en el estudio se tomen en cuenta la opinión de mujeres y de hombres con respecto a dichas creencias, permitirá explorar qué tan relacionadas están las creencias sobre masculinidad con aquellas sobre hombre maltratador, incorporando la perspectiva de género como una herramienta para el análisis de ellas. De acuerdo con todo lo anterior, esta investigación tiene como objetivos:

- Establecer las diferencias entre mujeres y hombres en cuanto a las creencias sobre masculinidad y hombre maltratador.

- Establecer la relación entre las creencias sobre masculinidad y las creencias sobre hombre maltratador.

Tomando en cuenta los objetivos planteados, se establecieron las siguientes hipótesis:

H1. Existen diferencias estadísticamente significativas entre mujeres y hombres en sus creencias sobre la masculinidad.

H2. Existen diferencias estadísticamente significativas entre mujeres y hombres en sus creencias sobre hombre maltratador.

H3. Existe correlación estadísticamente significativa entre las creencias sobre masculinidad y las creencias sobre hombre maltratador en hombres y mujeres.

Metodología

Participantes

Participaron en la investigación 200 mujeres y hombres, bajo un muestreo no probabilístico por cuota, 117 (58.5\%) mujeres y 83 (41.5\%) hombres. Estos sujetos, que colaboraron de manera voluntaria, fueron 
seleccionados de poblaciones de estudiantes de educación media superior, estudiantes universitarios y usuarios de Jurisdicciones Sanitarias de los Servicios de Salud del Estado de Yucatán, México, por su accesibilidad. En términos sociodemográficos: las edades fluctuaron entre 18 y 63 años, con una media de 25 años; participaron 173 (86.5\%) habitantes del área urbana y 27 (13.5\%) del área rural; y 134 (67.0\%) de los participantes eran solteros y $66(33.0 \%)$ eran casados.

Instrumentos y/o materiales

Las dos escalas utilizadas en esta investigación ya han sido validadas para poblaciones similares a la de este trabajo. Como se consigna en la descripción de cada una de ellas, ambas cuentan con una consistencia interna suficientemente buena para este tipo de instrumentos (Aiken, 1994) y en ambos casos también se explica más del 50\% de la varianza (Hair, Anderson, Tatham \& Black, 1999), lo cual implica una adecuada definición del constructo.

Escala de Creencias acerca de la Masculinidad (ECAM). Desarrollada por Gómez y Carrillo (2007) para medir las creencias que tienen las personas con respecto a la masculinidad, es una escala tipo Likert con cinco opciones pictográficas de respuesta que van de Totalmente de acuerdo a Totalmente en desacuerdo. Se encuentra conformada por 32 reactivos divididos en seis factores. La consistencia interna total de la escala fue de a .90 con un $57.86 \%$ de varianza total explicada.

Factor I. Discurso Social. Explora creencias acerca del imaginario social con respecto al significado de ser hombre, como el machismo, el ser mujeriego, el uso del poder y el ejercicio de la violencia por parte de los varones para controlar y dominar a las mujeres. Se integra por un total de 11 reactivos. Este factor presenta una consistencia interna de $\alpha .87$.

Factor II. Normativo. Explora creencias acerca de las reglas o códigos de comportamiento que se adjudican a un hombre, como el hecho 
de ser proveedores y ser la cabeza de las relaciones entre un hombre y una mujer. Se encuentra conformado por 5 reactivos. Muestra una consistencia interna de $\alpha .75$

Factor III. Iniciativa. Explora creencias relacionadas con el papel activo que debe tener el hombre en las relaciones con una mujer, la solución de problemas y el desempeño del hombre en el espacio público. Se integra por un total de 4 reactivos y muestra una consistencia interna de $\alpha .71$.

Factor IV. Superioridad. Explora las creencias que tienen que ver con la idea de que los hombres son superiores a las mujeres por el simple hecho de ser hombres. Consta de un total de 4 reactivos y una consistencia interna de $\alpha .71$.

Factor V. Imagen Externa. Explora las creencias con respecto a la apariencia física de un hombre y como ésta se relaciona con la masculinidad, así como el uso de la violencia como un atributo o acto de demostración del ser hombre. Este factor se estructura con 5 reactivos y presenta una consistencia interna de $\alpha .63$.

Factor VI. Honorabilidad. Explora las creencias que se tienen con respecto a los hombres como los responsables de resolver los problemas en la casa. Este factor está conformado por 3 reactivos y presenta una consistencia interna $\alpha .56$.

Escala de Creencias acerca del Hombre Maltratador (ECHOM). Desarrollada por Gómez, Carrillo y Revilla (2008) para medir las creencias que tienen las personas con respecto al hombre maltratador, es una escala tipo Likert con cinco opciones pictográficas de respuesta que van de Totalmente de acuerdo a Totalmente en desacuerdo. Está conformada por 15 reactivos divididos en tres factores. La consistencia interna total de la escala presenta un $\alpha .816$ y un $51.48 \%$ de varianza total explicada.

Factor I. Distante Emocional. Evalúa las creencias con respecto al hombre maltratador relacionadas con una estructura rígida de género, hace 
referencia al poco control de impulsos y al poco contacto de las emociones por parte de los hombres que ejercen violencia. Se encuentra conformado por 7 reactivos y presenta una consistencia interna de $\alpha .80$.

Factor II. Doble Fachada. Explora las creencias acerca del varón que ejerce violencia hacia las mujeres con respecto a la imagen que proyecta, en cuanto a sus conductas en el espacio público (trabajo, amigos, escuela) y en el espacio privado (hogar, relación de pareja, familia). Este factor se encuentra integrado por un total de 5 reactivos, con una consistencia interna de $\alpha .62$.

Factor III. Factores Asociados. Mide las creencias que las personas tienen con respecto al hombre maltratador que se relacionan con algún factor asociado para el ejercicio de la violencia, como puede ser la vivencia de violencia en la infancia, el uso y consumo de alguna droga, o haber ejercido violencia en alguna otra relación de vinculación afectiva. Dicho factor está constituido por 3 reactivos y presenta una consistencia interna de $\alpha .498$.

Cada una de las escalas contenía un apartado de datos sociodemográficos, con el cual se recopiló información referente a la edad, el sexo, el lugar de residencia, la escolaridad y el estado civil de las personas que formaron parte del estudio, variables que se utilizaron para coadyuvar en el análisis y la discusión de los datos obtenidos.

Tipo de estudio y diseño

Fue un estudio cuasiexperimental, ex post facto, transversal, de campo, de dos muestras independientes (Pick \& López, 2000), de tal manera que solo se hicieron inferencias de las relaciones existentes entre las variables, sin intervención directa, a partir de la variación concomitante de la variable independiente y las dependientes (Kerlinger, 2002). Asimismo, se utilizó un diseño correlacional para determinar la relación existente entre las variables de las creencias de la masculinidad y de las creencias acerca del hombre maltratador existentes de acuerdo con la variable sexo. 
Procedimiento

Se solicitaron los permisos pertinentes en los diferentes espacios donde se encontraban los participantes y se les solicitó su colaboración voluntaria. Posteriormente, se realizó la administración colectiva de los instrumentos a los y las participantes que conformaron la muestra bajo la siguiente instrucción:

El presente cuestionario forma parte de un trabajo de investigación, le pedimos por favor su colaboración para responderlo y le aseguramos que los datos serán analizados de manera anónima, confidencial y global, le agradecemos de antemano su participación y honestidad al contestar el cuestionario.

A continuación se le presentan una serie de afirmaciones, señale el cuadro que refleje qué tan de acuerdo está con la afirmación presentada; esto es, mientras más grande sea el cuadro que señale, quiere decir que está de acuerdo y mientras más chico sea, está en desacuerdo. Le pedimos que no deje de contestar ninguna afirmación y si tiene dudas hágasela saber al aplicador.

Para el análisis de los datos se utilizó un prueba t para establecer las diferencias hipotetizadas y la prueba de correlación Producto-momento de Pearson para establecer las relaciones entre las variables.

\section{Resultados}

Al realizar el análisis de medias sobre las creencias acerca de la masculinidad entre los grupos de hombres y mujeres, los resultados revelaron que hubo diferencias estadísticamente significativas en los seis factores que conforman la escala. Sin embargo, todas las medias encontradas para cada uno de los factores están por debajo de la media teórica (3.0). En general, las mujeres están más en desacuerdo en todos los factores, mientras que los hombres se encuentran en mayor acuerdo con las afirmaciones de los factores que componen la escala. Esta tendencia se presenta de nuevo al comparar la media general con las medias que presentan los hombres (ver Tabla 1). 
Tabla 1

Diferencias entre mujeres y hombres sobre las creencias acerca de la masculinidad

\begin{tabular}{|c|c|c|c|c|c|}
\hline $\begin{array}{c}\text { Factores de } \\
\text { Masculinidad }\end{array}$ & $\begin{array}{c}\text { Media } \\
\text { Mujeres }\end{array}$ & $\begin{array}{c}\text { Media } \\
\text { Hombres }\end{array}$ & $\begin{array}{c}\text { Media } \\
\text { Gral. }\end{array}$ & $t$ & $g l$ \\
\hline I. Discurso Social & 1.30 & 1.80 & 1.51 & $-6.3^{* * *}$ & 198 \\
II. Normativo & 1.84 & 2.51 & 2.12 & $-5.7^{* * *}$ & 198 \\
III. Iniciativa & 1.47 & 1.98 & 1.68 & $-5.4^{* * *}$ & 198 \\
IV. Superioridad & 1.63 & 2.24 & 1.88 & $-5.7^{* * *}$ & 198 \\
V. Imagen Externa & 1.77 & 2.13 & 1.92 & $-3.6^{* * *}$ & 198 \\
VI. Honorabilidad & 1.90 & 2.50 & 2.15 & $-3.4^{* * *}$ & 198 \\
\hline *** $<<.000$ &
\end{tabular}

Respecto al análisis de las diferencias entre hombres y mujeres en las creencias acerca del hombre maltratador, se encontraron diferencias estadísticamente significativas en dos de los tres factores que integran la escala. En el Factor II. Doble Fachada, las mujeres están en desacuerdo con respecto a que los hombres que maltratan presenten una doble fachada; y en el Factor III. Factores Asociados, las mujeres se encuentran en mayor desacuerdo que los varones con las afirmaciones respecto a estas creencias relativas al hombre maltratador. Asimismo, el Factor I. Distancia Emocional, si bien no presentó una diferencia estadísticamente significativa entre hombres y mujeres, es el único factor cuyas medias se encuentran por arriba de la media teórica (3.0) (ver Tabla 2). 
Tabla 2

Diferencias entre mujeres y hombres sobre las creencias acerca del hombre maltratador

\begin{tabular}{|c|c|c|c|c|c|c|}
\hline $\begin{array}{c}\text { Factores de } \\
\text { Hombre } \\
\text { Maltratador }\end{array}$ & $\begin{array}{c}\text { Media } \\
\text { Mujeres }\end{array}$ & $\begin{array}{c}\text { Media } \\
\text { Hombres }\end{array}$ & $\begin{array}{c}\text { Media } \\
\text { Gral. }\end{array}$ & $t$ & $g l$ & Prob. \\
\hline $\begin{array}{c}\text { I. } \\
\text { Distancia } \\
\text { Emocional }\end{array}$ & 3.87 & 3.77 & 3.83 & .93 & 156.13 & .335 \\
\hline $\begin{array}{c}\text { II. } \\
\text { Doble } \\
\text { Fachada }\end{array}$ & 1.55 & 2.06 & 1.76 & -5.75 & 198 & $.000^{* * *}$ \\
\hline $\begin{array}{c}\text { III. } \\
\text { Factores } \\
\text { Asociados }\end{array}$ & 1.56 & 2.20 & 1.83 & -5.58 & 198 & $.000^{* * *}$ \\
\hline
\end{tabular}

*** $\mathrm{p} \leq 0.001$

Por último, se realizó un análisis de asociación por medio del coeficiente de correlación Producto-momento de Pearson para conocer si existe relación entre las creencias sobre la masculinidad y las creencias sobre el hombre maltratador. A continuación se presentan los datos obtenidos de manera general (ver Tabla 3). Como se puede observar, el Factor I. Distancia Emocional no presenta correlaciones estadísticamente significativas con alguno de los factores de las creencias acerca de la masculinidad; sin embargo, todos los coeficientes de este factor son de carácter negativo, lo cual indica una relación inversa entre la distancia emocional y las creencias sobre masculinidad. Por otro lado, los Factores II y III de hombre maltratador se correlacionan significativa y positivamente con todos los factores de creencias sobre masculinidad, con correlaciones que van de medias altas a altas. 
Tabla 3

Correlación general entre creencias sobre masculinidad y hombre maltratador

\begin{tabular}{|c|c|c|c|}
\hline \multirow{2}{*}{$\begin{array}{c}\text { Factores de } \\
\text { Masculinidad }\end{array}$} & \multicolumn{3}{|c|}{ Factores de Hombre Maltratador } \\
\cline { 2 - 4 } & $\begin{array}{c}\text { I. Distancia } \\
\text { Emocional }\end{array}$ & $\begin{array}{c}\text { II. Doble } \\
\text { Fachada }\end{array}$ & $\begin{array}{c}\text { III. Factores } \\
\text { Asociados }\end{array}$ \\
\hline I. Discurso Social & -.127 & $.866^{* *}$ & $.757^{* *}$ \\
\hline II. Normativo & -.008 & $.857^{* *}$ & $.884^{* *}$ \\
\hline III. Iniciativa & -.092 & $.793^{* *}$ & $.807^{* *}$ \\
\hline IV. Superioridad & -.138 & $.685^{* *}$ & $.666^{* *}$ \\
\hline V. Imagen Externa & -.075 & $.621^{* *}$ & $.628^{* *}$ \\
\hline VI. Honorabilidad & -.043 & $.574^{* *}$ & $.618^{* *}$ \\
\hline
\end{tabular}

${ }^{* *} \mathrm{p} \leq 0.01$

Posteriormente, se realizó un análisis por sexo con el fin de establecer las diferencias en dichas concepciones entre mujeres y hombres. A continuación se presentan los resultados obtenidos del análisis de relación en mujeres. De acuerdo con esta información, el Factor I. Distancia Emocional, no presenta correlaciones estadísticamente significativas con alguno de los factores de las creencias sobre masculinidad, asimismo, todos los coeficientes son de carácter negativo a excepción del coeficiente entre la distancia emocional y el Factor II. Normativo. En el caso de los Factores II y III de hombre maltratador, ambos presentan correlaciones altas positivas con el Factor II. Normativo; correlaciones medias altas positivas con los factores correspondientes a Discurso Social e Iniciativa; por último, correlaciones medias bajas positivas con los factores correspondientes a Superioridad, Imagen Externa y Honorabilidad (Tabla 4). 
Tabla 4

Correlación entre creencias sobre masculinidad y hombre maltratador en mujeres

\begin{tabular}{|c|c|c|c|}
\hline \multirow{2}{*}{$\begin{array}{c}\text { Factores de } \\
\text { Masculinidad }\end{array}$} & \multicolumn{3}{|c|}{ Factores de Hombre Maltratador } \\
\cline { 2 - 4 } & $\begin{array}{c}\text { E. Distancia } \\
\text { Emocional }\end{array}$ & $\begin{array}{c}\text { II. Doble } \\
\text { Fachada }\end{array}$ & $\begin{array}{c}\text { III. Factores } \\
\text { Asociados }\end{array}$ \\
\hline I. Discurso Social & -.063 & $.653^{* *}$ & $.558^{* *}$ \\
\hline II. Normativo & .142 & $.781^{* *}$ & $.823^{* *}$ \\
\hline III. Iniciativa & -.014 & $.516^{* *}$ & $.640^{* *}$ \\
\hline IV. Superioridad & -.096 & $.367^{* *}$ & $.326^{* *}$ \\
\hline V. Imagen Externa & -.028 & $.314^{* *}$ & $.378^{* *}$ \\
\hline VI. Honorabilidad & -.067 & $.295^{* *}$ & $.408^{* *}$ \\
\hline
\end{tabular}

${ }^{* *} \mathrm{p} \leq 0.01$

Por otra parte, los resultados obtenidos del análisis de relación en hombres, muestran, al igual que las mujeres, que el Factor I. Distancia Emocional, no presenta correlaciones estadísticamente significativas con alguno de los factores de las creencias sobre masculinidad. Asimismo, todos los coeficientes son de carácter negativo a excepción del coeficiente entre la distancia emocional y el Factor VI. Honorabilidad. En el caso del Factor II. Doble Fachada, se encontraron correlaciones estadísticamente significativas altas y positivas con todos los factores de creencias sobre masculinidad, exceptuando el Factor VI. Honorabilidad, con un coeficiente de correlación medio alto positivo. Por último, el Factor III. Factores Asociados, presentó coeficientes de correlación estadísticamente significativos para todos los factores de creencias sobre masculinidad, mostrando una correlación alta positiva en los primeros cuatro y media alta para los dos últimos (ver Tabla 5). 


\section{Tabla 5}

Correlación entre creencias sobre masculinidad y hombre maltratador en hombres.

\begin{tabular}{|c|c|c|c|}
\hline \multirow{2}{*}{$\begin{array}{c}\text { Factores de } \\
\text { Masculinidad }\end{array}$} & \multicolumn{3}{|c|}{ Factores de Hombre Maltratador } \\
\cline { 2 - 4 } & $\begin{array}{c}\text { I. Distancia } \\
\text { Emocional }\end{array}$ & $\begin{array}{c}\text { II. Doble } \\
\text { Fachada }\end{array}$ & $\begin{array}{c}\text { III. Factores } \\
\text { Asociados }\end{array}$ \\
\hline I. Discurso social & -.139 & $.908^{* *}$ & $.786^{* *}$ \\
\hline II. Normativo & -.084 & $.874^{* *}$ & $.895^{* *}$ \\
\hline III. Iniciativa & -.110 & $.853^{* *}$ & $.842^{* *}$ \\
\hline IV. Superioridad & -.143 & $.750^{* *}$ & $.757^{* *}$ \\
\hline V. Imagen Externa & -.088 & $.775^{* *}$ & $.740^{* *}$ \\
\hline VI. Honorabilidad & .076 & $.669^{* *}$ & $.664^{* *}$ \\
\hline
\end{tabular}

$\mathrm{p} \leq 0.01$

\section{Discusión}

Con base en los resultados, se confirmó la Hipótesis 1 que establece diferencias entre hombres y mujeres en sus creencias sobre masculinidad; se confirmó parcialmente la Hipótesis 2 que establece las diferencias entre hombres y mujeres en sus creencias sobre hombre maltratador; y por último, se confirmó parcialmente la Hipótesis 3 que plantea la correlación entre las creencias sobre masculinidad y hombre maltratador en hombres y mujeres.

Respecto a las creencias acerca del guión o script (De Keijzer, 2005) que las y los yucatecos tienen acerca de la masculinidad, cuando se hace un análisis general de los datos, se obtienen las siguientes lecturas:

Primero, todas las puntuaciones se encuentran por debajo de la media teórica, lo cual indica que, en general, tanto hombres como mujeres participantes están en desacuerdo con la idea de la masculinidad hegemónica, donde el hombre es conceptualizado como el fuerte, el 
responsable de las cosas, activo, propositivo y resolutivo. Estos resultados concuerdan con los de Carrillo (2007), donde se puede percibir una especie de cambio o transición en el discurso del modelo hegemónico de la masculinidad-feminidad entre los hombres y mujeres de la sociedad yucateca, mostrando una especie de apertura en la concepción que se tiene del hombre y de la mujer.

En cuanto a las mujeres, los resultados indican que existe desacuerdo con las creencias acerca de la masculinidad que posicionan al hombre en un mayor nivel y estatus con respecto a ellas. El rechazo al modelo hegemónico de la masculinidad por parte de las mujeres puede explicarse en los costos que tiene para este grupo de la población. Todo esto deja en una voz muda el rechazo de las mujeres a este modelo hegemónico (Castro \& Riquer, 2004).

En cuanto a los hombres, los resultados indican que ellos están más de acuerdo con las creencias que se relacionan con la masculinidad hegemónica (Connel, 1995; De Keijzer, 2005; Garda 2006). Sin embargo, sus puntajes se encuentran por debajo de la media teórica, lo cual podría interpretarse como un aparente rechazo a la conceptualización de la masculinidad hegemónica. Esto puede explicarse de dos formas, la primera, como una etapa de transición donde el hombre se plantea más alternativas para el ejercicio de la masculinidad alejado del discurso social que tiene el prototipo y estereotipo de hombre dominador, que discrimina y subordina a otros hombres y mujeres (De Keijzer, 2006); y la segunda, es la del doble discurso o lo que Bonino (2002) llama micromachismos, donde el ejercicio del poder por parte del hombre en diferentes espacios es casi invisible.

En síntesis, si bien no todos los hombres se enmarcan o asumen esta conceptualización de la masculinidad - hegemónica- sí todos se benefician de las ganancias que ésta proporciona en el ámbito social (Castro \& Riquer, 2004). Esto provoca una percepción diferencial en un mismo constructo como es la masculinidad.

Respecto a las creencias acerca del hombre maltratador, los resultados obtenidos en el Factor I. Distancia Emocional no presentaron 
diferencias significativas entre mujeres y hombres, sin embargo, sus puntajes promedio se encuentran por arriba de la media teórica. Este primer factor (Distancia Emocional) conceptualiza al hombre maltratador como una persona que ejerce violencia porque tiene un pobre control de impulsos y una baja autoestima; esto es, ejerce violencia porque no puede "controlarse", no porque quiera hacerlo. En este sentido, se puede interpretar que la violencia de un hombre maltratador está vista como un componente interno y natural.

Por otro lado, se encontraron diferencias significativas entre mujeres y hombres en los otros dos factores que hablan de: (a) una doble fachada en el ejercicio de la violencia y (b) de factores asociados al ejercicio de la violencia como el uso de alcohol o experiencias tempranas de maltrato. Lo anterior sugiere que las mujeres creen que los hombres son violentos sin necesidad de aparentar socialmente y que dicha violencia no se debe a elementos externos como el alcohol, drogas o experiencia previa. Las mujeres de este estudio perciben que el ejercicio de la violencia por parte de los hombres se relaciona más con aspectos intrínsecos y poco con aspectos extrínsecos. Los datos se contraponen con los obtenidos por la ENVIM (2003), donde se exploran las percepciones de las mujeres respecto a las razones y causas de la violencia, encontrando que las razones que ellas expresan como causas, responden a una naturaleza externa, ajena a la voluntad del hombre violento. En ambos casos, dichas percepciones redundan en un fenómeno en el cual se desresponsabiliza al hombre de cualquier efecto que el ejercicio de la violencia pueda traer.

En contraposición, las creencias de los hombres se encuentran más de acuerdo con la idea de que el hombre maltratador, si bien presenta una dificultad para controlar sus emociones, se debe a que tiene un factor asociado como el consumo de alguna droga y, de la misma manera, presenta una doble fachada. Esto es, que el hombre maltratador no lo es por naturaleza, sino porque hay factores extrínsecos que lo hacen ser violento.

En ambos casos, tanto mujeres como hombres expresan un aparente desacuerdo con las creencias acerca del hombre maltratador asociadas con 
factores externos y a mostrar una doble fachada. Desde esta perspectiva hay acciones de los varones que las mujeres no consideran o identifican como violencia, sino como parte de un comportamiento natural e incuestionable acorde a su condición de varones (Ramírez, 2006). También existe cierto tipo de violencia que se considera legítima e incluso deseable, que implica una connotación disciplinaria; son acciones que son percibidas como justas para la sociedad donde se ejerce (ENVIM, 2003). De la misma manera, la percepción de lo que es la violencia en algunas ocasiones se confunde con rasgos de la personalidad y carácter del individuo. En otras palabras, se argumentan como "formas de ser", apelando a la esencia natural de la persona, por lo que se asume la imposición como natural, incluso como una forma de expresión afectiva por parte del hombre hacia la mujer, percepción que es compartida por hombres y mujeres (Ramírez \& Vargas, 1998).

Respecto a las correlaciones de las creencias acerca de la masculinidad con las del hombre maltratador, se observan correlaciones de todos los factores de las creencias de la masculinidad con dos de los tres factores de las creencias acerca del hombre maltratador. Lo cual sugiere que la percepción de masculinidad se relaciona, al menos parcialmente, con el ejercicio de la violencia. Por otro lado, el único factor que no correlaciona significativamente con las creencias sobre masculinidad es el relacionado con la distancia emocional que presenta el hombre maltratador; sin embargo, es importante señalar que todos los coeficientes presentan una direccionalidad negativa. En este sentido, tanto mujeres como hombres parecen estar de acuerdo que el terreno emocional no es terreno de lo masculino (De Keijzer, 2006; Castro \& Riquer, 2004; Carrillo, 2007).

En el caso de las mujeres, las correlaciones más altas son aquellas que se relacionan con las ideas o códigos que se adjudican a los hombres como ser proveedor, cabeza de familia, etc. Las correlaciones medias altas se relacionan con la construcción social de que ser hombre significa ser macho y/o mujeriego, y con las creencias de que los hombres deben ejercer un papel activo en las relaciones interpersonales, en la solución de problemas en el espacio público. Por último, las correlaciones medias bajas son aque- 
llas que se relacionan con las creencias de que los hombres son superiores a las mujeres; del vínculo de la apariencia física y masculinidad, la necesidad de ser violentos para demostrar su masculinidad; así como su responsabilidad de resolver problemas en la casa. Estos datos permiten afirmar que las percepciones de las mujeres con respecto a la violencia que ejerce el hombre es un problema que involucra, además del vínculo de pareja, un conjunto complejo de relaciones con el entorno social que favorecen la aparición de la violencia, la perpetúan o contribuyen a su solución.

En el caso de los hombres, las correlaciones más altas son aquellas que se relacionan con las creencias o códigos adjudicados a un hombre como ser proveedor y cabeza de familia; tener un papel activo en su relación con las mujeres y la solución de problemas en el espacio público; ser machista, mujeriego y dominante; ser superior a las mujeres por el simple hecho de ser hombre; y tener una apariencia física masculina, así como usar la violencia para demostrar masculinidad. Las correlaciones medias altas implican que el hombre es el responsable de la solución de los problemas en la casa. En este sentido, los resultados concuerdan con los de Ramírez (2003) donde los hombres perciben su violencia como parte de la identidad masculina. Asimismo, en el caso de los hombres, es primordial la influencia de lo social y colabora en una construcción de género dirigida hacia el comportamiento violento.

En síntesis, los resultados muestran que las mujeres manifiestan que socialmente se tolera la violencia del hombre. Por su parte, los hombres relacionan más la masculinidad con el ejercicio de la violencia, sus creencias tienden a vincular el ser violentos con factores extrínsecos; en este sentido, lo peligroso de esta postura radica en que ni la mujer ni el hombre responsabilizan al hombre que ejerce violencia de sus actos, sino que lo justifican como parte del rol social que le toca interpretar en la sociedad (ENVIM, 2003; Ramírez, 2006). De acuerdo con esto, se puede decir que el género estructura los discursos de tal manera que se mantiene el status quo de la desigualdad, aunque se manifieste un desacuerdo entre hombres y mujeres. Cabe señalar que los roles sociales que presentan un carácter rígido generan menos posibilidades de desarrollo de proyectos individuales y colectivos (Ramírez, 2006). 
Los resultados de este trabajo concuerdan con los obtenidos por González (1998), que incluyó a mujeres y hombres, donde se pone de manifiesto la existencia de una percepción diferencial de la violencia entre mujeres y hombres, fenómeno que se explica por los procesos de construcción simbólica de cada género en particular. Esto pone de manifiesto que los significados, las valoraciones de éstos y otras acciones, objetos y relaciones, son observados desde una posición filtrada por el género (Ramírez, 2006). La subjetividad es el punto de referencia y abre la posibilidad a explicaciones diferenciales ante un mismo fenómeno, como se ha observado en estudios específicos donde participan hombres (Garda, 2006; Ramírez, 2003).

La información que proporcionan los hombres no solo sirve para conocer la relación que tienen la masculinidad y la violencia, como un mero ejercicio de exploración del discurso de género, sino que permite fortalecer y mejorar el impacto de las intervenciones con hombres, así como, el desarrollo de la teoría respecto a las masculinidades y el ejercicio de la violencia. Es evidente que los estudios de las masculinidades se encuentran en desarrollo desde diferentes disciplinas y con ópticas diversas; sin embargo, resulta imperativo que los hombres se involucren en el desarrollo de la teoría de la masculinidad teniendo como herramienta de análisis la perspectiva de género y el cuestionamiento al poder; que los estudios no exalten la diferencia en un discurso explicativo circular donde las mujeres son diferentes por el simple hecho de ser mujeres y del mismo modo con los hombres; y visibilizar que hombres y mujeres no son entes aislados, sino en mutua interrelación. Hoy más que nunca es necesario generar discursos alternativos de masculinidad-feminidad, para que la hegemonía no se convierta en una cárcel que impida a hombres y mujeres vivir en libertad, plenitud y con un verdadero acceso a una vida libre de violencia.

Aunque los resultados de esta investigación arrojan datos que enriquecen el diálogo en torno a los discursos sociales sobre la violencia y la masculinidad, es importante tomar en cuenta algunas limitaciones que se presentaron en este estudio. En primer lugar, no se pueden generalizar estos resultados a la población rural, dado que la muestra de estos participantes 
no es representativa de dicho grupo. En segundo lugar, en este trabajo solamente se abordaron las creencias sobre la masculinidad hegemónica, por lo tanto, resulta importante considerar modelos alternativos de las masculinidades emergentes. Por último, tampoco se logró representatividad por grupos etarios, lo cual no permitió establecer las diferencias que podrían presentarse entre éstos.

En conclusión, tanto mujeres como hombres rechazan la idea de una masculinidad hegemónica, lo cual puede indicar la gestación de un cambio en la construcción del significado de masculinidad. Dicho cambio representa mayores dificultades en la reconstrucción de un significado para los hombres, considerando el costo que esto les implica.

Por otro lado, aunque sin el sustento de la significancia, se observó una tendencia en los participantes - mujeres y hombres - a justificar el comportamiento violento de un hombre maltratador como algo interno e incontrolable, lo cual estaría justificando, a su vez, el ejercicio de la violencia al considerarla como algo propio y natural de ser hombre.

Sin embargo, una creencia que sí establece una actitud diferencial entre mujeres y hombres es que para ellas se mantiene el argumento de lo intrínseco como explicatorio de la violencia; en tanto que para ellos, el comportamiento violento está asociado con factores externos como el consumo del alcohol, drogas o experiencias tempranas de maltrato. Como consecuencia, para las mujeres, al naturalizar la violencia, no existirían posibilidades de cambio y para los hombres este comportamiento es justificado por factores que no dependen de ellos; en ambos casos estas creencias contribuyen a mantener un círculo de violencia.

Para finalizar, las creencias sobre masculinidad y hombre maltratador, tanto de las mujeres como de los hombres, constituyen una red de conductas, cogniciones y afectos que sostienen el ejercicio de la violencia. De esta manera, mientras el imaginario social esté regido por una necesidad de proactividad e instrumentalidad en los hombres, la violencia de género continuará presentándose en nuestra sociedad. 


\section{Referencias}

Aiken, L. R. (1994). Psychologycal testing and assessment. (Eighth edition). Boston: Allyn and Bacon.

Bonino, L. (2002). Develando los micromachismos en la vida conyugal. En J. Corsi et al., Violencia masculina en la pareja. Una aproximación al diagnóstico y a los modelos de intervención. (pp. 191-208). Buenos Aires, Argentina: Paidós.

Carrillo Trujillo, C. (2007). Masculinidad-Feminidad: buscando una identidad. En T. Castillo y L. Cortés (Eds.), Psicología Social en Yucatán: avances y prospectiva (pp. 191-206). Mérida, Yucatán: Universidad Autónoma de Yucatán.

Castro, R. \& Riquer, F. (Coords.) (2004). Violencia de género entre las parejas mexicanas. Resultados de la Encuesta Nacional sobre la Dinámica de las Relaciones en los Hogares 2003, México D.F.: Instituto Nacional de las Mujeres.

Connel, D. (1995). Organización social de la Masculinidad, Masculinidades. Berkeley: California Press.

Contreras Urbina, J. (2005). El riesgo de ejercer violencia física contra la pareja: cuándo y por qué. Hallazgos de un estudio con varones trabajadores en la Ciudad de México. Género y Salud en Cifras, 2(4), 21-26.

Corral, P. (2000). Violencia contra la mujer. Debats, (70/71), 94-102.

De Keijzer, B. (2005). Masculinidades y Violencia de Género. En M. Elu y E. Santos (Eds.), A lo largo del camino (pp. 217-226). México: Programa de las Naciones Unidas para el Desarrollo.

De Keijzer, B. (2006). Hasta que el cuerpo aguante: género, cuerpo y salud masculina. La manzana, 1(1), 59-88.

ENDIREH (2003) Encuesta de la Dinámica de las Relaciones en los Hogares. Un acercamiento estadistico al problema de la violencia intrafamiliar en Yucatán. Mérida: Instituto para la Equidad de Género en Yucatán.

Garda, R. (2006). La violencia masculina en la relación de pareja en la Ciudad de México. Análisis desde la perspectiva de género y de la estructuración. En R. Garda y F. Huerta (Comps.), Estudios sobre la violencia masculina (pp. 243-297). Ciudad de México: IndesolHombres por la Equidad A. C.

Garda, R. (2007). Aqui por lo menos yo toco pared, y está muy dura... Mujeres, hombres e Instituciones ante el trabajo con la violencia masculina en el hogar. Documento interno. Ciudad de México: Hombres por la Equidad A. C. 
Gómez, J. \& Carrillo, C. (2007). Validación de una escala de Masculinidad en Yucatecos [Resumen]. Memorias del XXXIV Congreso del Consejo Nacional para la Enseñanza e Investigación en Psicología, CNEIP, Guadalajara, México.

Gómez, J., Carrillo, C. \& Revilla, J. (2008). Validación de una escala sobre creencias del hombre maltratador en mujeres y hombres Yucatecos [Artículo]. Memorias del IV Congreso Internacional de Psicología Social, Benemérita Universidad Autónoma de Puebla, México.

González, S. (1998). Los silencios de la salud reproductiva, violencia, sexualidad y derechos reproductivos. México: Asociación Mexicana de Población y la Fundación John D. y Catherine T. Mac Arthur.

Hair, J.F. Anderson, R. E., Tatham, R. L. \& Black, W. C. (1999). Análisis multivariante. (5a edición). Madrid: Prentice Hall.

Kerlinger, F. (2002). Psicología experimental. México: McGraw-Hill.

Lamas, M. (1997). La perspectiva de género: una herramienta para construir la equidad entre mujeres y hombres. México: Desarrollo Integral de la Familia.

Organización Mundial de la Salud. (2003). Informe Mundial sobre la Violencia y la Salud. Washington, DC., OPS/OMS.

Pick, S \& López, S. (2000). Cómo investigar en ciencias sociales. México: Trillas.

Ramírez, J. (2003). Masculinidad y violencia doméstica. Guadalajara: Centro de Investigaciones y Estudios Superiores en Antropología Social Occidente.

Ramírez, J. (2006). La violencia de los varones contra sus parejas heterosexuales: realidades y desafíos. Un recuento de la producción mexicana. En Salud Pública de México, 48(2), 315-327.

Ramírez, J. \& Vargas, P. (1998). La cifra negra de la violencia doméstica contra la mujer. En Los Silencios de la Salud reproductiva: violencia, sexualidady derechos reproductivos (pp. 107-133). México: Asociación Mexicana de Estudios Parlamentarios.

Secretaría de Salud (2003). Encuesta Nacional sobre Violencia contra las Mujeres (ENVIM). México, D.F.: Instituto Nacional de Salud Pública.

Fecha de recepción: 13 de mayo de 2010.

Fecha de aceptación: 13 de diciembre de 2010. 